\title{
„Scheinbare Extravaganz". \\ Pagane und christliche Platoniker über die Wunderlichkeit des Mythos
}

\begin{abstract}
It seems that the Duchess of Somerset has been going into some Board School somewhere where the children were taught fairy-tales, and then going into some Board of Guardians somewhere else and saying that fairy-tales were full of ,nonsense, “ and that it would be much better to teach them about Julius Caesar „or other great men.“ Here we have a complete incapacity to distinguish between the normal and eternal and the abnormal or accidental.
\end{abstract}

(G. K. Chesterton, The Illustrated London News, Dec $2^{\text {nd }}, 1905$ )

\section{Einleitung}

In den ersten „christlichen Jahrhunderten“ des Römischen Reiches ist die platonische Philosophie sicherlich einer der geistigen Orte, an denen sich die quantitative Präsenz von und der qualitative Umgang mit antiken Mythen am intensivsten belegen lassen. Dabei gilt es allerdings, „Platonismus“ als Synekdoche zu verstehen und zwischen dem Platonismus als philosophischer Einzelströmung und dem Platonismus als allgemeiner Denkform zu unterscheiden: Als allgemeine Denkform hatte der Platonismus in vielen, vielleicht sogar in nahezu allen Bereichen den Status eines gegenüber den Einzelauffassungen von der Welt relativ unabhängigen diskursindifferenten Ausdrucksmediums gewonnen, und so lässt sich feststellen:

Mit einer Aussage „... war Platoniker“ ist von etwa 220 n. Chr. an sehr wenig gewonnen. Der Platonismus gewann - fast schlagartig - eine erhebliche Breitenwirkung. Platonische Fachsprache wurde zum Vehikel nahezu jeder philosophischen Diskussion. Aber auch die absichtlich dunklen Offenbarungen der hermetischen Schriften „platonisieren“. Es dürfte schwer sein, ProsaAutoren [...] des 3., 4. und 5. Jahrhunderts zu benennen, die nicht irgendwie eine Einwirkung des Platonismus erkennen lassen. ${ }^{1}$

Zu den so - das heißt: sensu lato - verstandenen „Platonikern“ gehörten auch christliche Denker, die gegen Einzelaussagen der platonischen Philosophenschule im engeren Wortverständnis argumentierten und Einspruch erhoben. Folgendes soll nun Ziel meiner Erörterung sein: Zu zeigen, dass der Umgang mit den Mythen zu den Bereichen gehört, in denen dieses platonisch gedachte Argumentieren gegen schulmäßig Platonisches besonders deutlich wird und vor allem, wie der pagane Platonismus auf die damit entstehende Herausforderung reagiert. Und so wird die Tatsache, dass von den christlichen Platonikern mit den Bibelerzählungen mutatis mutandis nach dem Muster der paganen

1 Heinrich Dörrie: Die platonische Philosophie des Kelsos in ihrer Auseinandersetzung mit der christlichen Theologie. Göttingen 1967, S. 50. 
platonischen Mythendeutung umgegangen wird, den Hintergrund für das Verständnis der Polemik bilden müssen, die sich zwischen christlichen und paganen Platonikern über die Einzelheiten des Umgangs mit dem paganen Mythos entspinnt. Bestenfalls wird sich nach Sichtung einiger aussagekräftiger Beispielbelege dazu ein systematisches Fazit ziehen lassen. Begonnen sei mit einem Blick auf den für uns als historische Gestalt ziemlich rätselhaften Neuplatoniker Sal(l)ustios und seine konzise Programmatik des neuplatonischen Mythenverständnisses. ${ }^{2}$

\section{Die átoпía des Mythos als Abwegigkeit: Sallust}

Zwischen den Jahren 361 und 363 entsteht, wohl auf Anregung Kaiser Julians II. Apostata - und als theoretisches Instrument seines neuplatonisch durchtränkten Revitalisierungsprogramms der paganen Religiosität, eine kleine Schrift mit dem Titel Über

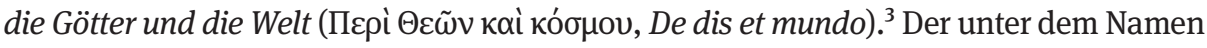
Sal(l)ustios bekannte Autor dieses „platonischen Katechismus“, ${ }_{4}$ erklärt in einem bemerkenswerten Abschnitt seiner handbuchartigen Lehrschrift, dass an der Wahrheit der alten Mythen trotz ihrer „scheinbaren Abwegigkeit“ oder „scheinbaren Extravaganz“ festgehalten werden sollte. Das entscheidende Textstück darf als Aufgriff traditioneller Themen der Auseinandersetzung mit den Vorwürfen christlicher Denker gegen die paganen Mythen gelten, aber auch als theoretische Selbstvergewisserung der platonischen Denktradition in dieser Angelegenheit. Sallust führt als Erklärung für seine Haltung in dieser Frage zunächst ein Effizienzargument ad verecundiam an (De dis et mundo III 1), wie es ähnlich des Öfteren in der antiken Literatur auftaucht, insbesondere wenn es um die Frage des Vertrauens in Orakel geht: Dass die Mythen zweifellos göttlichen Ursprungs sind, zeige sich daran, wie die größten der Dichter und die besten der Philosophen

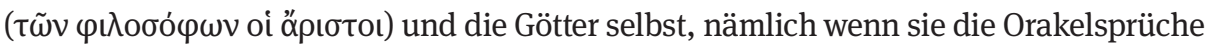

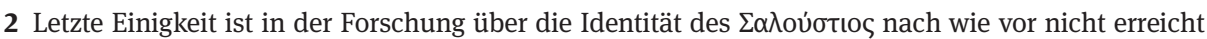
worden. Die beiden gehandelten Kandidaten sind: Saturninius Secundus Salustius, Berater des Kaisers und von Julian II. zum Prätorianerpräfekten des Ostens ernannt (PLRE I 814-817), und Flavius Sallustius, Prätorianerpräfekt von Gallien und 363 Konsul mit Julian (PLRE I 797 f.; vgl. 796). Von Saturninius Secundus Salustius ist philosophische Betätigung überliefert, die Indizien scheinen also bis auf Weiteres insbesondere für ihn zu sprechen.

3 Textgrundlage für den Traktat ist die Ausgabe von Gabriel Rochefort (Hg.): Saloustios, Des dieux et du monde. Paris ${ }^{2} 1983$; zum Vergleich der Interpretations- und Übersetzungsvarianten oft interessant: Arthur Darby Nock (Hg.): Sallustius, Concerning the Gods and the Universe. Cambridge 1926, sowie: Enrique Ángel Ramos Jurado (Hg.): Salustio: Sobre los dioses y el universo. Madrid 1989, S. 266-316. 4 Als ein solcher ist das Werk wohl tatsächlich zu verstehen. Zur Katechismusform platonischer Handbücher (nicht nur) im vierten Jahrhundert vgl. Christian Schäfer: Julian Apostata und die philosophische Reaktion gegen das Christentum: Die „Pseudomorphosen“ des platonischen Denkens im „magischen Zeitalter“. In: ders. (Hg.): Kaiser Julian ,Apostata“ und die philosophische Reaktion gegen das Christentum. Berlin 2008, S. 41-64. Zur „Restauration“ der alten Religion unter Julian vgl. auch Theresa Nesselrath: Kaiser Julian und die Repaganisierung des Reiches. Konzept und Vorbilder. Münster 2013. 
formulierten, von der mythischen Ausdrucksform Gebrauch gemacht hätten. Warum dies auch in Anbetracht der Abwegigkeit der bunten Mythenwelt so als richtig und zustimmungswürdig zu erachten ist, bezeichnet Sallust anschließend als ein Beantwortungsproblem, das der Philosophie obliegt. Die Antwort der Philosophie aber laute folgendermaßen: Nach dem simile simili-Prinzip gleiche doch alles seinem Ursprung.

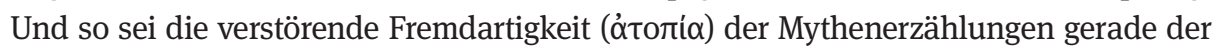
beste Hinweis darauf, dass die Mythen göttlich sind ( $\theta \varepsilon \tilde{o}$ o oi $\mu$ ṽ $\theta$ o $)$. Gleichen die Mythengeschichten doch gerade darin den Göttern, dass sie so seltsam bekannt und gleichzeitig unbekannt wirken, so vertraut menschlich und doch so verstörend nichtmenschlich, so offensichtlich überall und doch so geheimnisvoll, so präsent und gleichzeitig so wirklichkeitsenthoben, so einfach überzeugend und gleichzeitig so rea-

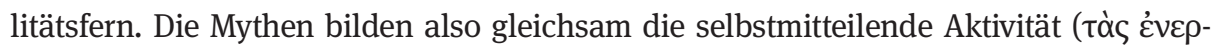
ycíac) der Götter ab, wie sie insbesondere bekanntermaßen in ihrer sich im Offensichtlichen verbergenden Ausdrucksweise in den Orakeln regelrecht „zur Sprache kommt“ (und insbesondere in diesem Punkt erfolgt der Aufgriff der langen Tradition der Orakelapologetik bei Sallust).

An diese Stelle (De dis et mundo III 3) schließt sich unmittelbar der berühmte Ausspruch des Autors an, eigentlich könne in diesem Sinne ja auch die ganze Welt

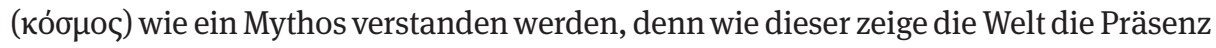
des Göttlichen sowohl in der offenkundig sinnlich zugänglichen Verfassung des wahrnehmbar körperlichen Universums als auch in der darin verhüllt uns sinnlich

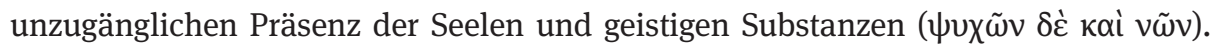
Weltbau, Mythos und Orakel sind nur verschiedene Weisen der Selbstmitteilung des Göttlichen: Alle drei sind semantisch (im etymologischen Sinn von $\sigma \tilde{\eta} \mu \alpha$ als greifbar äußeres Zeichen, sichtbare Bezeichnung) verschiedene, doch inhaltsgleiche Ausdrucksweisen der göttlichen (Selbst)Äußerung. Die Art dieser Äußerung aber ist adressatenspezifisch menschengemäß - in Geschichten und sichtbaren Dingen. Das Problem ist: Das bloß Menschliche kongruiert nicht mit dem Göttlichen, und das zeigt auf, wie die Weise des Gesagten danach verlangt, dass dem Inhalt des Gemeinten nachgespürt werde.

Dies einmal festgesetzt, stellt sich Sallust dem wohl traditionellsten Vorwurf gegen die Mythen (De dis et mundo III 4) und wirft die Frage auf, warum die mythischen Erzählungen in so auffälliger Häufung moralisch Anstößiges, ja teilweise sogar Abartiges von den Götter erzählen: Ehebruch, gegenseitiges Bestehlen und Gewalt gegen die Eltern etwa - ein stets wiederkehrender Kritikpunkt seit Xenophanes von Kolophon, ${ }^{5}$ aufgenommen und radikalisiert in der Dichterkritik Platons in der Politeia ${ }^{6}$ und von der christlichen Polemik gegen die pagane Religion. Gilt also auch für diese An-

5 Vgl. bei Xenophanes vor allem die Fragmente 21 B $10-12$.

6 So in den beiden großen Dichterkritiken im zweiten (379a-398b) und im zehnten Buch der Politeia (595a-608b). Eine gute Bestandsaufnahme bietet Stephen Halliwell: The Republic's Two Critiques of Poetry. In: O. Höffe (Hg.): Platon, Politeia. Berlin 1997, S. 313-332. 
stößigkeiten die Dialektik von Verhüllen und Enthüllen, wie das für den Kosmos als ganzen oder die Orakelsprüche so anzunehmen ist? In den Worten von Sallust:

Ist denn auch all dies der Bewunderung würdig, weil es die Seele sogleich darauf bringt, dank der scheinbaren Extravaganz das Gesprochene wie einen Schleier und die Wahrheit als unaussprechlich anzunehmen? ${ }^{7}$

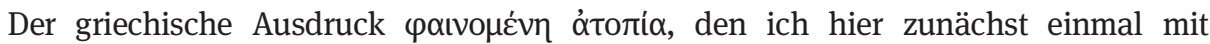
„scheinbare Extravaganz“ übersetzt habe, kann auf mehrerlei Weise verstanden werden. Sallust scheint damit, d.h. mit der Charakterisierung dieser „Extravaganz“ als paıvo$\mu \varepsilon ́ v \eta$, erstens sagen zu wollen, man müsse das Zugeständnis machen, dass diese ótoтí $\alpha$ einem geradezu ins Gesicht springt und tatsächlich verstört. Zweitens aber besagt der

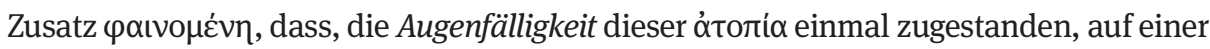
zweiten Auffassungsebene eher die Augenscheinlichkeit als das hermeneutische Erklärmoment der richtigen Mythenauffassung zu gelten habe.

Anders als das christliche Realsymbol, das die im Miterleben der Augenzeugen belegte Konkretisierung des Logos im einmaligen Einzelfall der Geschichte bedeutet, ist das Mythische in seinen Geschichten nicht historisch und im Einzelfall miterlebbar. ${ }^{8}$ Sallust folgt hier der platonischen Deutungslinie, wie sie etwa von Plutarch vorgeben wurde. ${ }^{9}$ Doch im Anwendungsschluss ist Sallust deutlicher und fordernder als Plutarch: Das im Mythos Gesagte ist Vermittlung und will erschlossen statt bezeugt werden. So kommt Sallust zu der wohl berühmtesten Aussage seines Traktats und gleichzeitig zu einer der vielleicht treffendsten Aussagen, die ein antiker Autor jemals über den Mythos und die Dinge, die er erzählt, formuliert hat:

Diese Dinge sind nie passiert, doch sie geschehen fortwährend. Was überzeitliches Denken als ein „zugleich“ von allem wahrnimmt, zerlegt der Diskurs menschlichen Denkens in ein zeitliches Nacheinander. ${ }^{10}$

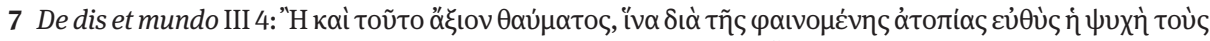

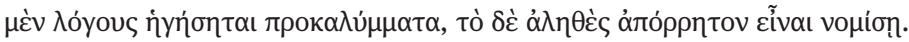

8 Anders als das Vertretungssymbol ist das Realsymbol ein hinweisendes Sinnbild, in dem die bezeichnete Wirklichkeit selbst schon in realer Weise präsent ist -,,it intrinsically constitutes what it signifies“ (Stephen M. Fields: Being as Symbol. Washington, D.C. 2000, S. 24). Dagegen haben beide Arten des Symbolischen den Anspruch, eine nicht als gegenwärtig fassbare Realität als gegenwärtig erscheinen zu lassen.

9 Vgl. Plut. mor. 25C. Zur Deutung ist u. a. hilfreich: Maren Niehoff: Philo and Plutarch on Homer. In: dies. (Hg.): Homer and the Bible in the Eyes of Ancient Interpreters. Leiden/Boston 2012, S. 127-153, v. a. S. 139.

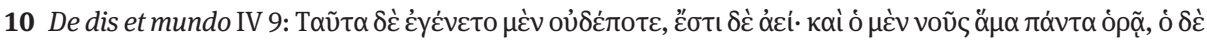

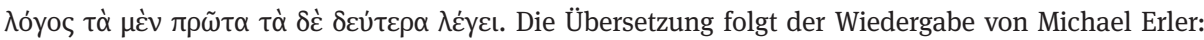
Praesens divinum. In: Markus Janka/Christian Schäfer (Hg.): Platon als Mythologe. Darmstadt 22014, S. 61-78, hier S. 67. 
Wären die Dinge, die in den alten Mythen erzählt werden, auch in dieser Weise historisch geschehen, wären sie nach Sallusts Dafürhalten tatsächlich skandalös. Doch sind sie so eben nicht geschehen, sie sind vielmehr die narrative Verschlüsselung ewiger welterklärender Abläufe in einer dem Menschen gemäßen Sprachgestalt. Von der Güte der Götter ist gerade im Umfeld Kaiser Julians oft die Rede, da es ja unter anderem darum gehen muss, auch in dieser Hinsicht ein ebenbürtiges oder besseres Gegenprogramm zum Christentum zu propagieren. Diese Güte der Götter äußert sich in der neuplatonischen Programmatik der paganen Autoren nun aber vor allem darin, sich secundum modum recipientis mitzuteilen, und das hat gleichzeitig eine pädagogische Funktion für den menschlichen Adressaten - eine wichtige Erklärfigur in der neuplatonischen Theologie. ${ }^{11}$

Auf dieser Grundlage unterscheidet Sallust vier Arten, oder eher Auffassungsweisen des Mythos, und er macht sie deutlich am Einzelbeispiel des Mythos von Kronos, der seine Kinder verschlingt:

Die Auffassung als theologische Mythen ( $\theta \varepsilon$ co入оуıкоi), die tatsächlich anagogischen Charakter haben und etwas Bedeutsames über das Göttliche aussagen, wenn auch vielleicht nur insofern, als es im Menschen wirkt und die Seele verstehen lässt, was das Göttliche in ihr oder an ihr ist: So zeigt die Geschichte vom Vater, der sich sein eigen Fleisch und Blut einverleibt, bildhaft die Reflexion des Geistes, der im Ausgang von sich selbst die Weltwirklichkeit durchgeht, um in sich selbst zurückzukehren; - die „physische“ ( sichtbaren Weltbau zeigt, wie Kronos, aufgefasst als Chronos, die Eigenart der Zeit lehrt, das von ihr Hervorgebrachte auch wieder zu verschlingen;

- die psychischen Auffassungsarten ( $\psi$ vхккоi), die im narrativen Bild vom nachwuchsverschluckenden Vater erklären, wie Gedanken als von der menschlichen Geisttätigkeit hervorgebrachte trotzdem in dieser Geisttätigkeit verbleiben;

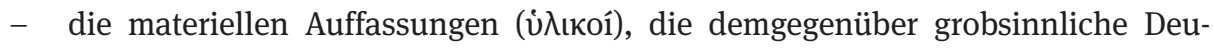

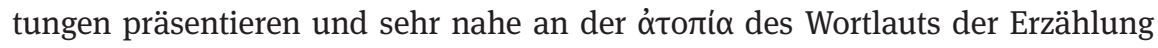
bleiben: Sallust schmäht an dieser Stelle die Ägypter dafür, dass sie mit ihrer Darstellung der Götter mit Tierleibern oder Tierhäuptern dieser Auffassungsweise nahestehen.

Keine dieser Auffassungsweisen, auch nicht die materiell identifizierende, nimmt die mythische Erzählung allerdings primär als einmalige Geschichte, die sich so zeitlich lokalisierbar tatsächlich, historisch ereignet hat.

Während mit dem historischen Faktum alles erfüllt zu sein scheint und die „Fülle der Zeit“ dann ein für allemal für sich steht, verlangen die verschlüsselten unzeitlich gültigen mythischen Geschichten nach einer erklärenden Entschlüsselung - darin Märchen vergleichbar, die man Kindern erzählt, nicht, um sie zu lehren, dass es die

11 So bei Plot. enn. VI 9[9]3,37-54. Vgl. dazu ergänzend die Ausführungen z. B. bei Pierre Hadot: Wege zur Weisheit. Frankfurt 1999 (frz. 1995), S. 195. 
„atopischen“ Schreckgestalten wie Drachen, Hexen und andere Geschäftsführer des Bösen wirklich gibt, sondern um sie zu lehren, dass das Böse in all seinen Auftretensgestalten überwunden werden kann, wie es in einem berühmten Ausspruch Chestertons heißt. ${ }^{12}$ Auch diese bunten und teilweise befremdlichen Märchen sollen ja den Auftakt dazu geben, zu einer reifen Bestimmung von Gut und Böse zu führen und im Loslassen der Geschichten zur Überzeugung zu gelangen, es lohne sich, für das Gute einzustehen. Damit bewegt sich Sallust gänzlich innerhalb des Rahmens, den die platonische Tradition vorgibt. ${ }^{13}$

Sallust kontrastiert in diesem Zusammenhang (De dis et mundo III 3) offenbar bewusst ein aus dem Christentum bekanntes Zugangsprinzip, das sich vielleicht am bündigsten mit einem Hinweis auf ein Bibelzitat anzeigen lässt: „Vater, ich danke dir, dass du das den Klugen und Weisen verborgen hast, den Kleinen aber offenbart hast“ (Mt 11,25). Für Sallust kommt es mit der platonischen Allgemeinauffassung offenbar im Gegenteil darauf an, dasjenige, was den im Geiste Kleinen immer hinter einem Getöse von Extravaganzen verschlüsselt bleiben wird, durch Geistesübung zu entschlüsseln. Der Wortlaut der Mythenerzählungen hat es dann sozusagen mit dem Christentum gemeinsam, „Platonismus fürs Volk“ zu sein, nicht aber der Sinn der Mythen: Der erschließt sich der philosophischen Askesis, dem Abstreichen des Bildlichen und dem Lösen von den Phänomenen, wie es der Schulplatonismus lehren will und wie es den Weisen vorbehalten bleibt. (Das wiederum zeigt, wie weit der Platonismus des vierten Jahrhunderts vom antiken religiösen Denken entfernt steht, wo das Bild häufig genug schlicht das Gemeinte ist. ${ }^{14}$ Es ist interessant zu sehen, wie Sallust im Bestreben, die christliche Denkfigur vom Realsymbol zu vermeiden, damit in recht „unantike“ Deutungsmuster verfällt. Spätere Platoniker werden das - teilweise zumindest und unter vielen Mühen - zu korrigieren haben. ${ }^{15}$ ) Das alles soll nach Sallusts Ansicht das Bildliche freilich nicht gänzlich annullieren. Aber es zeigt, wie das Göttliche im Konkreten der plastischen Wirklichkeit von Welt und Mythos „vermummt“ ist. Ähnlich, wie Thomas Mann es beschreibt:

12 „Fairy-tales do not give the child the idea of the evil or the ugly; that is in the child already, because it is in the world already. Fairy-tales do not give a child his first idea of bogy. What fairy-tales give the child is his first clear idea of the possible defeat of bogy. The baby has known the dragon intimately ever since he had an imagination. What the fairy-tale provides for him is a St. George to kill the dragon." (G. K. Chesterton: Tremendous Trifles. New York 2007, S. 49).

13 Vgl. z. B. die wohl aus der Wende vom zweiten zum dritten Jahrhundert stammende Schrift des Pseudo-Plutarch De Homero (II) 92. Dort wird gesagt, der Mythos habe zwar den Charakter eines Rätsels (alvvy $\mu \alpha$ ), aber darin sei angesichts der pädagogischen Absicht dahinter nichts Sonderbares

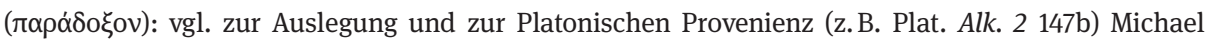
Hillgruber: Die Pseudoplutarchische Schrift De Homero, Teil 2. Stuttgart/Leipzig 1999, S. 211.

14 Vgl. dazu u.a. Clifford Ando: The Matter of the Gods. Religion and the Roman Empire. Berkeley/Los Angeles/London 2008, dort insbesondere das Belegbeispiel auf S. 22-27.

15 Vgl. dazu z.B. Harold Tarrant: Literal and Deeper Meanings in Platonic Myths. In: Catherine Collobert/Pierre Destrée/Francisco J. Gonzalez: Plato and Myth. Studies on the Use and Status of Platonic Myths. Leiden/Boston 2012, S. 47-66. 
Gleichzeitigkeit ist die Natur und Seinsart aller Dinge, ineinander vermummt erscheinen die Wirklichkeiten, und nicht weniger ist ein Bettler ein Bettler, weil möglicherweise ein Gott sich in ihn verstellt. Ist nicht ein Strom ein Gott, von Stiergestalt oder auch von der eines bekränzten Mannweibs mit doppelartiger Brust, hat er das Land nicht geschaffen, und nährt er es nicht? Das hindert nicht ein sachliches Verhalten zu seinem Wasser, nüchtern gleich diesem: man trinkt's, man befährt es, man wäscht sein Leinen darin, und nur das Wohlgefühl, das man empfindet beim Trinken und Baden, mag einer Mahnung an höhere Gesichtspunkte gleichkommen. Zwischen Irdischem und Himmlischem ist die Grenze fließend, und nur ruhen zu lassen brauchst du dein Auge auf einer Erscheinung, damit es sich breche ins Doppelgesichtige. ${ }^{16}$

Positiv konfrontieren lässt sich dieser Gedanke bei Sallust mit einer Stelle aus der wohl an die Wende des zweiten zum dritten Jahrhundert zu verweisenden pseudo-plutarchischen Schrift De Homero ([II] 92), die für diese Tradition der Mythenauffassung so etwas wie einen Schlüsselcharakter zu haben scheint. Ähnlich wie bei Sallust herrscht hier

der Gedanke vor, daß die in der Dichtung verborgene Wahrheit mit Hilfe des Mythos vor falschen Interpretationen durch die Masse geschützt werden kann; nur die Lernbegierigen dringen zu ihr vor und werden durch die mythische Verkleidung zusätzlich zum Nachdenken angespornt, während die Unverständigen gar nicht erst in Versuchung geraten, die Geheimnisse zu entweihen, sondern sich an der äußeren Hülle erfreuen. ${ }^{17}$

Doch lässt sich die scheinbare, oberflächliche, offenkundige oder vermeintliche ó тоті́ $\alpha$ des Mythischen eben noch in anderen Schattierungen auffassen und erklären als bei Sallust. Im Folgenden soll daher der Versuch unternommen sein, die átoтí des Mythischen nach möglichen Bedeutungsvarianten zu entschlüsseln, die sich allesamt bei platonischen Autoren finden lassen, um dadurch diese verschiedenen Varianten mit verschiedenen platonischen Denkern in Verbindung zu bringen, bei denen sich diese áтоті́ $\alpha$ sozusagen dingfest machen lässt. Das Hauptaugenmerk wird dabei auf stichprobenartig ausgesuchten Autoren des vierten Jahrhunderts liegen und auf dem bei Sallust formulierten Gedanken der vorsätzlich befremdenden Abwegigkeit der Mythenbilder, denn das dürfte nach redlichem Ermessen die einende Hauptthese der paganen philosophischen Auffassungen im vierten Jahrhundert darstellen. Den Anfang machen soll allerdings ein Blick auf Platon. In Platons Auffassung vom Mythenerzählen finden sich nämlich die Versatzstücke der Erklärung bei Sallust allesamt präformiert die pädagogische Entfremdung genauso wie die gezielte Provokation des Denkens, die Bildwerttheorie genauso wie der anagogische Sinn der Mythen.

16 Thomas Mann: Joseph und seine Brüder, Bd. 2. Frankfurt a.M. 1986, S. 902.

17 Hillgruber (s. Anm. 13), S. 212, übrigens explizit mit Verweis auf De dis et mundo III 4. Vgl. zusätzlich zum „Hintersinn“ der Mythen in den spätantiken platonischen Deutungen Reinhold Merkelbach: Isis regina - Zeus Serapis. Stuttgart/Leipzig 1995, S. 335-339. 


\section{Die átoría des Mythos als Kuriosität: Platon}

Für Platons Philosophie ist die Dialogform charakteristisch und sie ist auch für die inhaltliche Deutung der platonischen Werke von kaum zu überschätzendem Belang. ${ }^{18}$ Dass in den Dialogen auch monologische Passagen von Mythenerzählungen auftauchen, ist somit nicht so ohne Weiteres zu erwarten. Auffällig ist dabei allenthalben, dass jeder Platonische Mythos traditionelle mythische Versatzstücke oder Strukturen integriert. Und das mit Absicht: Mit einem Mythos will Platon offenbar immer etwas Bekanntes anklingen lassen, da das Mythische dazu dient, über geläufige Motive Neues oder Außerordentliches, das der Dialog erbracht hatte, narrativ nahe zu bringen. Dieses Verfahren ist dem Platonischen Mythos mit der Einführung neuer Bedeutungen für überkommene „normalsprachliche“ Wörter durch Platon in den Dialogen gemeinsam. ${ }^{19}$

Warum das bei Platons Mythenerzählungen so ist, lässt sich wohl vor allem mit folgendem Hinweis erklären: Eines der identifizierenden Kennzeichen ${ }^{20}$ der Mythen bei Platon ist, dass sie bevorzugt am Beginn oder am Ende einer dialektischen Dialogpassage stehen und somit ihre psychologische oder pädagogische Stoßrichtung offenbaren. Der Mythos gibt Anlass zu einer argumentativ begründenden Erörterung oder schließt eine solche ab, etwa, um ihre Ergebnisse emotional zu festigen. Der Mythos ist somit zum guten Teil auch wie eine captatio benevolentiae über das Hilfsmittel des kulturell Vertrauten zu verstehen, als das Einbringen einer „heimeligen“ Motivik aus bekannten Mythenthemen.

Aus den solcherart dialogdienlichen Charakteristika der Platonischen Mythen lassen sich nun verschiedene durchaus positive Wirkungen in der Absicht des Mythenerzählens ersehen, die Jean Pépin als „objektive“ und „subjektive Wohltaten“ (bienfaits) oder „Dienste“ der Platonischen Mythen folgendermaßen zusammengefasst hat: ${ }^{21}$

Auf der „objektiven Seite“ steigert oder erschließt der Platonische Mythos in der bildlichen Wiederholung des in der Argumentation Gewonnenen den Bedeutungsreichtum einer Aussage durch vielfache Auslegungsmöglichkeiten; er erleichtert die Analyse und Darstellung eines komplexen Problems durch den Appell an Intuition, visuelles Vorstellen und Ähnliches; und er respektiert und kennzeichnet Tabufelder

18 Für die folgenden Überlegungen vgl. auch Christian Schäfer: Art. „Mythos/Mythenkritik“. In: Chr. Horn/J. Müller/J. Söder (Hg.): Platon-Handbuch. Stuttgart 2009, S. 209-213.

19 Vgl. zu diesem Vorgehen beispielhaft Karl Kerényi: Griechische Grundbegriffe. Zürich 1964, S. $12-$

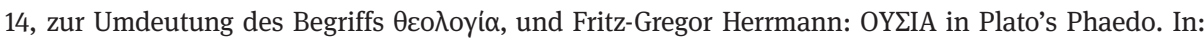
ders. (Hg.): New Essays on Plato. Language and Thought in Fourth-Century Greek Philosophy. Swansea 2006, S. 43-73, vor allem S. 44-59, zur Neudeutung von oủoía.

20 Vgl. hierzu die Merkmalssammlung des platonischen Mythos bei Glenn W. Most: Platons Exoterische Mythen. In: M. Janka/Chr. Schäfer (Hg.): Platon als Mythologe. Neue Interpretationen zu den Mythen in Platons Dialogen. Darmstadt 22014, S. 9-21, 13-15.

21 Vgl. Jean Pépin: Mythe et allégorie. Paris 1972, S. 479-482. 
und Grauzonen von Themenbereichen, indem er in Bildern davon redet. Das also ist der Effekt der kulturellen Oikeiosis des Gedankens, seiner funktionalen „Heimeligkeit".22

Von der „subjektiv wohltuenden“ Seite her betrachtet ist es mit dieser zunächst gezielt eingesetzten Heimeligkeit allerdings schnell vorbei. Der Mythos nämlich entfaltet beim Hörer eine kinetische Energie, er stimuliert zum Weiterdenken, und das oft vor allem durch seine prima facie absurd anmutenden oder kuriosen Darstellungselemente, seine ótoríaı also - so sagt es Platon deutlich im Phaidros (229e): Die mythische Rede von Chimären und Pegasen und anderen Kuriositäten, átoríaı (,Verlegenheiten“ übersetzt Ernst Heitsch ${ }^{23}$ ), dieser Art verweise den Menschen doch letztlich auf sich selbst zurück. Denn bevor man sich die Frage stellen dürfe, ob diese mythischen ótođía alle wahr seien, müsse man sich ja genau genommen erst einmal darauf besinnen, ob der Mensch in seiner Bauart und seinen Möglichkeiten denn nicht ein viel kurioseres Wesen sei als all diese Mythengestalten oder sogar der phantastisch unwahrscheinliche Typhon in den mythischen Urgeschichten. Diese Phaidros-Stelle ist im Übrigen auch deswegen für den Zusammenhang mit Sallust so interessant, weil sie die ótoтía der Mythen durch das explizite Zitat des delphischen Aufrufs „Erkenne dich selbst“ mit dem Orakelwesen in Verbindung bringt.

Der Platonische Mythos nimmt somit den philosophischen Theorieübungen des Dialogs, die er kontrastiert, bildhaft zusammenfasst oder widerspiegelt, die Langeweile und Trockenheit, womit er gleichzeitig den Dialog auflockert und durch den gezielten Einsatz der kuriosen Erzählmomente mit Spannung lädt; und er ist dank dieser kuriosen Denkanstöße gleichsam ein hermeneutischer Belastungstest, wie eine Nagelprobe zur Aussonderung oder Unterscheidung derer, die sich auf Denken und Argumentation des Autors - oder zumindest: des Sprechers im Dialog - einlassen wollen oder nicht. ${ }^{24}$

Beide bei Sallust vermerkten Seiten des Mythischen sind also bei Platon deutlich da und erklären sich aus der pädagogischen Funktion, die Platon im Mythos sieht und die er deswegen in seinen Dialogen auch forçieren möchte: Das narrativ Zugängliche als Zugangserleichterung und Appell an die Vorstellungskraft und gleichzeitig das motivisch Verstörende als pädagogischer Anreiz, als Denkanstoß für diejenigen, die weiterdenken können und wollen. Wichtig ist vor allem der zweite Aspekt, da er im Weiteren eine Erklärung für die taktische Verwendung der óto $\boldsymbol{i}^{\alpha} \alpha$ eröffnet: Denn diese ist wie gesagt zu

22 Ähnlich lautet das Fazit zum Er-Mythos der Politeia bei Stephen Halliwell: The Life-and-Death Journey of the Soul: Interpreting the Myth of Er. In: G. R. F. Ferrari (Hg.): The Cambridge Companion to Plato's Republic. Cambridge 2007, S. 445-473: „tests the limits of understanding“, „yields a surplus of possible meanings that cannot be adequately encompassed by any simple interpretation“, ,stands in a kind of challenging counterpoint [...] with the rest of the Republic" (S. 445).

23 Platon, Phaidros. Übersetzung und Kommentar von Ernst Heitsch (Platon, Werke Bd. III,4), Göttingen 1993, ad locum.

24 Ähnliches zeigt die Sammlung Platonischer Selbstaussagen zu den verschiedenen Mythen bei Geneviève Droz: Les mythes platoniciens. Paris 1992, S. 15. 
verstehen als ein pädagogischer oder hermeneutischer Belastungstest. Wenn Kurt Flaschs augenzwinkernde Behauptung stimmt, der Platonismus habe nie für sich in Anspruch genommen, eine „Philosophie des gesunden Menschenverstands“ zu sein,, ${ }^{25}$ dann ist die $\alpha$ толі́ $\alpha$ des Mythischen bei Platon gerade sehr platonisch: Sie bewirkt eine Herausnahme des Hörers oder Lesers aus dem Üblichen und dem common sense des alltäglich Begegnenden, sie stört ihn förmlich auf und wird somit zum Anfang des Nachdenkens. ${ }^{26}$

Platon selbst gesteht die ó Teilen bewusst in einen traditionellen Rahmen gesetzten - Mythen an verschiedenen

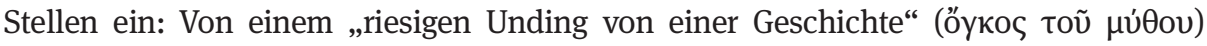
spricht er etwa im Politikos (277d) und entschuldigt sich für die konventionsbrechende Sperrigkeit der Erzählung; ${ }^{27}$ seine Dialogfigur Phaidros lässt Platon (Phaidr. 275b) anmerken, Sokrates habe die erstaunliche Gabe, ganz wunderliche „ägyptische“ und noch fremdartigere Mythen jederzeit wie bestellt aus dem Ärmel schütteln zu können; und im Gorgias (523a) muss Sokrates den Kallikles als Einleitung zum Vortrag seines Jenseitsmythos vorwarnen: „Was ich dir jetzt erzähle, wird dir wie ein abwegiges Mär-

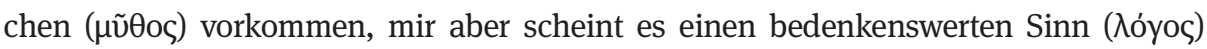
auszudrücken.“

25 So Kurt Flasch im Vorwort zu ders. (Hg.): Parusia. Studien zur Philosophie Platons und zur Problemgeschichte des Platonismus. Festgabe für Johannes Hirschberger. Frankfurt a.M. 1965.

26 Man sollte nicht übersehen, dass damit ein bedeutender Aspekt des traditionellen Mythenerzählens noch eingefangen wird, nämlich derjenige der Herausnahme aus der Gegebenheit des Normalen in zeitlicher Hinsicht: Zwar ist der platonische Mythos (soweit man weiß) nicht mehr Mythos in seiner Funktionalität als rituelle Vergegenwärtigung und er wird deswegen wohl auch keine Umsetzung in gottesdienstliche „Handlungszitate“ gekannt haben. Doch immerhin lässt sich auch vom platonischen Mythos behaupten: Als Mythos ist er in seiner eigenen Art und Weise anti-,,säkular“, da er den Hörer gezielt aus den Problemlagen des Saeculums - der Weltzeit - herausholt und, wenn doch nicht festlich, so doch geistig erhebend in Distanz zum solchermaßen aufgefasst Säkularen tritt. Platons Mythos erfüllt damit als Teil der platonischen Weltsicht die Diagnose, die Mircea Eliade dem Phänomen „Mythos“ in einer seiner heraushebenswerten kulturellen Transformationen ausstellt: Mythologie drängt in einigen Kulturen dazu, so Eliade, statt die ewige rituelle Wiederkehr in kosmischen Perioden - wie rituell vergegenwärtigend wirkenden Jahrestagen der erzählten Ereignisse - vorzugeben und mit Inhalt zu füllen, das Ewige als das dem Rhythmus der Wiederkehr Enthobene oder diesen Rhythmus Überdachende anzupeilen und sich damit der „Weltzeit“ zu entziehen, sie zu übersteigen oder in irgendeinem Sinne obsolet zu machen (vgl. Mircea Eliade: Ewige Bilder und Sinnbilder. Frankfurt a.M. 1998, S.65 - 68). In dem Maße, wie auf Platons Mythen Hans-Georg Gadamers Verdikt tatsächlich zutreffen sollte, dass sie nicht die „unenträtselte Gewißheit“ des religiösen Weltbezugs, sondern „das ins Kosmische ausgezogene Lineament der sich im Logos deutenden Seele“ zum Thema haben, wird Eliades Diagnose also womöglich zutreffen, und in dem Maße, wie der spätere Platonismus die Mythen als beides affirmiert sehen wollte, zum entsprechenden Teil ebenfalls (vgl. Hans-Georg Gadamer: Plato und die Dichter. In: ders.: Gesammelte Werke 5. Tübingen 1985, S.187-211; das Zitat findet sich auf S. 208). Zu einem Beispiel der rituellem Auffassung der Mythen im Platonismus vgl. auch unten Anm. 44.

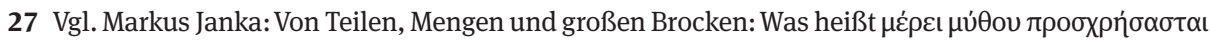
in Platons Politikos? In: RhM 145 (2002), S. 186-197. 
Die Platonische Theorie der Provokationsleistung des Mythenerzählens mit kuriosen Verstörelementen löste nun eine lange Tradition der Auseinandersetzung mit Sinn und Wert des Mythischen in der Philosophie aus. Sallust gehört in diese Tradition. Aber auch andere, von denen jetzt noch in stichprobenartiger Auswahl die Rede sein soll.

\section{3. '́xоті́ $\alpha$ als Befremdlichkeit und Unanständigkeit: Plutarch, Kelsos und Origenes}

Der (sit venia verbo) „Topos der átoтía“ spielt auch eine maßgebliche Rolle in der Diskussion um die allegorische Mythendeutung. Ganz im Sinne der von Platon vorgezeichneten heilsam verstörenden Funktion des Mythos und der von Sallust ins Auge gefassten psychagogischen Funktion dieser Verstörung spricht etwa Maximos von Tyros $(4,6 a)$ am Ende des zweiten Jahrhunderts von den anagogischen Wirkungen der Mythen als „,anleitende Handreichungen“ ( $\chi \varepsilon\llcorner\rho \alpha y \omega y i \alpha$ ist der hier verwendete Fachterminus der platonischen Terminologie) ${ }^{28}$, welche die Seele in ihrer Auslegungsarbeit an Text und

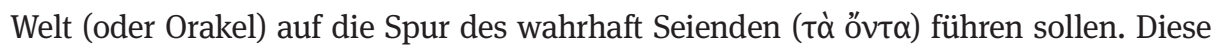
platonische Allgemeinauffassung hält sich erstaunlich invariant über die Jahrhunderte durch und lässt sich an der Wende vom vierten zum fünften Jahrhundert bei Augustinus belegen, wenn er ganz platonisch sagt, die dunklen Worte des Bibeltextes bildeten eine stets neue Herausforderung an den Verstand, transponierend den richtigen Sinn hervorzuholen. Auch hier steht das Motiv der „Hintersinns“, der erst hervorgeholt werden will, und der heilsamen Wirkung dieser Hintersinnsprovokation für den Verstand, der dadurch zur Tätigkeit angeregt wird, im Zentrum der Aufmerksamkeit. ${ }^{29}$

Plutarch berichtet nun, dass die Alten als den mit Mühe hervorzuführenden „Hintersinn“ (úróvoı $\alpha$ ) genau das benannten, was später mit dem terminus technicus $\alpha \lambda \lambda$ пуорí $\alpha$ bezeichnet wurde. ${ }^{30}$ Dieser unterschwellige Hintersinn (oder eher tragende „Untersinn“) der บ்ió-voı $\alpha$ ist in der mythischen Erzählung selbst nicht offen präsent und

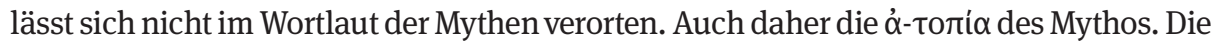
Befremdlichkeit des Wortlauts kann im Sinne eines Alteritätsmoments dann als effizienter Ursprung der Allegorese als hermeneutischem Prinzip gelten, die á zeichnet aber bemerkenswerterweise auch gleichzeitig die Grenze der Allegorisierbarkeit, und das ist nun besonders interessant:

28 Diese $\chi \varepsilon\llcorner\rho \alpha y \omega y i ́ \alpha$ ist typisch platonische Ausdrucksweise. Sie wird genauso von christlichen Denkern verwendet: Vgl. Wiebke-Marie Stock: Theurgisches Denken. Zur Kirchlichen Hierarchie des Dionysius Areopagita. Berlin 2008, S. 171-196. Für den Text des Maximos von Tyros folge ich der Übersetzung: Maximos von Tyros, Philosophische Vorträge. Übersetzt von Otto und Eva Schönberger. Würzburg 2001. 29 Vgl. Aug. doctr. chr. II 6,7: quod totum provisum esse divinitus non dubito ad edomandam labore superbiam et intellectum a fastidio revocandum, cui facile investigata plerumque vilescunt. Zu dieser Stelle, dem Zitat des Maximos und dem in der Spätantike bekannten Motiv, Autoren drückten sich bewusst und gezielt unklar aus, vgl. auch Hillgruber (s. Anm. 13), S. 213.

30 Plut. mor. 19E (De audiendis poetis 4). Dazu einmal mehr Hillgruber (s. Anm. 13), S. 213. 
Diese Grenze sahen die Platoniker nämlich auch dort, wo zum Beispiel die konkurrierende stoische Schule in der Allegorisierung alter Mythen weitgehend bedenkenlos vorging. Während die Stoiker ihre Mühe darauf verwendeten, die Mythen auf das „physikalische“ Erklären des Zusammenkommens der weltkonstitutiven Elemente zu zentrieren, ${ }^{31}$ blieben die Platoniker bei der Platonischen Grundüberzeugung, der Mythos müsse ein moralisches und vor allem psychagogisches Potential haben und selbst dort, wo man den Stoikern Recht zu geben habe, dass hier in bunten Abfolgeerzählungen von der Bauart der Welt die Rede ist, müsse diese Bauart in der narrativen Darbietung einen kosmo-logischen, also in der Welterkenntnis sinnstiftenden und somit moralisch anleitenden Wert ${ }^{32}$ aufweisen - ganz so wie bei Platons kosmologischen Mythen, die allesamt ein „psychozentrisches Weltbild“33 malten. Tatsächlich sind uns Fälle überliefert, in denen sich die Platoniker gegen die Stoiker dort absetzen, wo die ó толí $\alpha$ der Mythen als allzu plastisch den moralischen Sinn des Mythenerzählens überhaupt gefährdet, zu verdecken droht oder gänzlich ad absurdum führt. ${ }^{34}$ Es gibt für die paganen

31 Vgl. dazu das Schlusskapitel des stoischen Mythenhandbuchs des Lucius Annaeus Cornutus (nat. 35, 13): „In dieser Weise, mein junger Schüler, solltest du nun schon auch das übrige, was über die Götter in mythischer Form überliefert zu sein scheint, nach dem hier dargebotenen Vorbild auf die aufgezeigten Grundelemente beziehen können; sei dabei überzeugt, dass die Früheren nicht die ersten besten waren, sondern dass sie fähig waren, die Natur des Kosmos zu verstehen, allerdings dazu neigten, sich über sie in Symbolen (symbola) und Rätseln (ainigmata) philosophisch zu äußern“ (Lucius Annaeus Cornutus, Einführung in die griechische Götterlehre, hgg., eingeleitet und übersetzt von Peter Busch und Jürgen K. Zangenberg. Darmstadt 2010). Ohne allzu stark zu vereinfachen lässt sich tatsächlich behaupten, dass die stoische Allegorese sich darum drehte, ,die traditionellen Götter in Eigenschaften, Teile und Vorgänge der physischen Welt komplett aufzulösen, jene durch diese restlos zu ersetzen“ (Fabio Berdozzo: Götter, Mythen, Philosophen. Lukian und die paganen Göttervorstellungen seiner Zeit. Berlin 2011, S. 181). Gleichermaßen interessant wie verwirrend ist vor diesem Hintergrund, dass Eusebius von Caesarea, die Schrift des Porphyrius Adversus Christianos zitierend, überliefert, der Lieblingsschüler Plotins habe dem Origenes unterstellt, er sei bei den Stoikern Chairemon und Cornutus für die allegorische Methode in die Schule gegangen, die er dann auf die Bibelexegese anwendete (Eus. h.e. 6,19,8, GCS 9,2, S. 560). Die Leitlinie für die platonische Kritik an der stoischen Methode der Allegorisierung gab Plutarch vor, insbesondere in seinen Ausführungen mor. 19E-20B (De audiendis poetis 4). Vgl. dazu u. a. Niehoff (s. Anm. 9), S. 136-137.

32 Zur Unterscheidung von Kosmographie und Kosmologie in der Philosophie der Antike, wobei jene sinnneutral als „Plan oder Beschreibung der Welt [...] in ihrer Struktur, ihren etwaigen Abstufungen, regionalen Unterschieden, usw.“ anzusehen ist, diese aber als eine Weltdarstellung, die unter Einrechnung des Menschen eine sinngebende Bedeutung des Kosmos eröffnet, vgl. Rémi Brague: Die Weisheit der Welt - Kosmos und Welterfahrung im westlichen Denken. München 2005 (frz. 1999), S. 12 und S. 14.

33 Vgl. David Sedley: Teleology and Myth in the Phaedo. In: Proceedings of the Boston Area Colloquium in Ancient Philosophy 5 (1989), S. 359-383, insbesondere S. 373.

34 Dabei kam diese Art der Allegorisierung ursprünglich als eine der dort als möglich gehandelten Auslegungsvarianten des Mythos offenbar aus der Schule Platons selbst und wurde von den Stoikern dann übernommen. Aus der Testimonienlage ergibt sich, dass insbesondere Xenokrates, der zweite Nachfolger Platons in der Akademie, hier eine richtungsweisende Rolle gespielt hat. Vgl. dazu John Dillon: The Heirs of Plato. A Study of the Old Academy (347-274 BC). Oxford 2003, S. 154. 
wie für die christlichen Platoniker eine auch für jede Allegorisierung unüberbrückbare Anstößigkeit des Mythos.

Es ist in diesem Zusammenhang von nicht zu unterschätzender Bedeutung, wenn Origenes gegen Kelsos in folgendem Fall „platonisch“ Stellung bezieht (Cels. IV 48): Im Heiligtum der Hera in Argos - nach anderen Quellen in Samos - gab es (offenbar) eine Darstellung der Göttin, wie sie den Göttervater Zeus oral befriedigte. Wie auch immer der ursprüngliche Sinn der Darstellung gewesen und welche Geschichte auch immer hier verarbeitet gewesen sein mag: Die Stoiker hatten den Aussagen der Origenes-KelsosDebatte nach anscheinend weder Bedenken noch Mühe, dieses Bildnis als kosmogo-

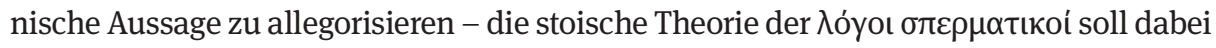
eine gewisse Rolle gespielt haben. ${ }^{35}$ Nun scheint Kelsos dieser Auslegung irgendwie Sympathien entgegengebracht $\mathrm{zu}$ haben oder doch zumindest bei Origenes in Verdacht zu stehen, diese stoische Auslegung zu verteidigen oder Verständnis für sie aufzubringen. Origenes für seinen Teil wendet sich nun erstens gegen die Darstellung selbst, da sie das Wesen des Göttlichen profaniere, und zweitens gegen den Versuch, diese Profanation auch noch durch die allegorische Zuweisung eines höheren - und sei es auch nur „physikalisch“ allegorisierenden - Sinns zu adeln und akzeptabel machen zu wollen. Die erste Stoßrichtung ist klar gegen die alten griechischen Religiositätsausdrücke gerichtet und will das Christentum als frei von solcherlei (!) Unanständigkeiten in der Gottesdarstellung erweisen; die zweite Stoßrichtung ist eigentlich keine christliche, sondern eine genuin platonische: ${ }^{36}$ Was die alte Religion an inakzeptablen Anstößigkeiten aufweise, dürfe auch nicht in der Menschengemeinschaft (bei Platon: der Polis)

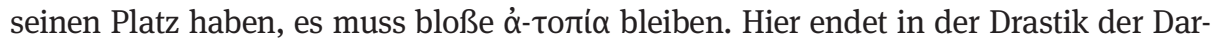
stellung die effektive Möglichkeit, dem Mythos einen psychagogischen Sinn und ein moralisches Entfaltungspotential zuzuweisen und ihn für ein für beide Seiten würdiges Gott-Mensch-Verhältnis in Anspruch zu nehmen. Die Polemik des Origenes gegen Kelsos erweist sich somit als genialer Kunstgriff, wobei es dem Platoniker Origenes zustatten

35 Vgl. Marianne Wifstrand Schiebe: Chrysippos und das obszöne Bild von Zeus und Hera. Eine forschungskritische Sichtung der Evidenz. In: Mnemosyne 65 (2012), S. 469-479; Robert Lamberton: Homer the Theologian. Neoplatonist Allegorical Reading and the Growth of the Epic Tradition. Berkeley 1986, S. 210; Maarten Jozef Vermaseren: Studies in Hellenistic Religions. Leiden 1979, S. 218.

36 Korrekter ausgedrückt ist weder die erste ausschließlich oder auch nur eindeutig „,christlich“ noch die zweite ausschließlich oder eindeutig „platonisch“: Wie noch des Näheren zu sehen sein wird, ist es gerade ein Anliegen auch und insbesondere der Platoniker, bestimmte Praktiken und Darstellungsformen des Religiösen philosophisch zu kritisieren und gewissermaßen ,aufzuheben“; andererseits ist es ein Zug, den das Christentum mit dem Platonismus und der gewichtigen Mehrheit der antiken Philosophenschulen gemein hat, dass auch religiöse Formen und Vorstellungen, die nicht zum eigenen Sinnsystem gehören, als ernstzunehmende Quelle von Wahrheiten und Weisheit verstanden werden im Sinne des Christentums also etwa als eine praeparatio evangelii. Damit teilen Platonismus und Christentum wie in so vielen anderen Dingen einen Grundzug, der für das Verhältnis von Denksystemen oder -schulen ein Charakteristikum der Zeit seit etwa dem ersten Jahrhundert vor Christus bezüglich der Einschätzung religiöser Praktiken und „Weisheitslehren“ darstellt: Vgl. dazu die einschlägige Studie von Peter van Nuffelen: Rethinking the Gods. Philosophical Readings of Religion in the Post-Hellenistic Period. Cambridge 2011. 
kam, dass der Platoniker Kelsos offenbar den Fehler begangen hatte, sich irgendwie auf die Seite der stoischen Allegorese der fraglichen Darstellung zu stellen oder ihr Sympathien entgegenzubringen: ${ }^{37}$ Der Christ Origenes konnte sich anhand der umstrittenen Deutung eines paganen Mythos mit seiner Position als der bessere Platoniker erweisen als Kelsos, jedenfalls aber konnte er sich für pagane Platoniker als annehmbarer erweisen als ein paganer Stoiker.

An solchen drastischen Beispielen und anhand der hermeneutischen Verzweiflungstat, sich in der Verteidigung der mythischen Götterdarstellung schließlich vielleicht sogar solchen stoischen Allegorisierungen an die Brust zu werfen, lässt sich auch verdeutlichen, wie hart im Platonismus das Ringen darum war, den Wortsinn und die plastische Darstellungsweise des Mythos ernstzunehmen und sich nicht einfach darüber hinwegzusetzen. Zumindest was die Mythen Platons betraf, war es platonische communis opinio, dass beides, Wortsinn und Materialsinn, bis zu gewissen Punkten stets widerspruchsfrei Hand in Hand gehen könne. Auch hier findet der christliche Platonismus ganz ähnliche Wege wie der pagane. So gehört der Platoniker Augustinus zu den Bibelexegeten, die den mehrfachen Wortsinn des Bibeltextes dadurch verteidigen, dass sie jede der Sinnstufen, angefangen von der wortwörtlichen Auffassung, als mit den anderen vereinbar ansehen, auch wenn die elaborierteren Sinnstufen stets den Verständnisschlüssel zum Literalsinn bilden können.

So gilt für die im Platonismus mit Hingabe und immer besserer Ausarbeitung betriebene Orientierung am Hintersinn und der Erschließungsarbeit, die das Wesentliche der Aussage nicht im Text, sondern „hinter“ dem Text sieht, Ähnliches wie für die philosophische Weltdeutung. Diese war in der Antike früh an den Punkt gelangt, der den Aufruf nötig werden ließ, die Erscheinungen über der Hintergründigkeitsarbeit in der Wirklichkeitserklärung nicht gänzlich zu vergessen, „die Erscheinungen zu retten“, wie

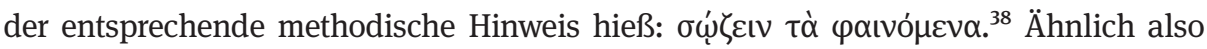
musste die Beschäftigung mit dem Mythos immer wieder zu dem Methodenhinweis zurückkommen, den Wortsinn nicht aufzugeben, sondern ihn in der Erklärungsarbeit zu „retten“.

37 Einem Beitrag aus dem Auditorium der Frankfurter Tagung folgend sei diese Bestandsaufnahme der Position des Origenes noch durch folgenden Hinweis ergänzt: Origenes hat an anderer Stelle auch mit den óтоті́丿ı der Bibelgeschichten gerungen und sie von Fall zu Fall wegen ihrer Abwegigkeit als nicht mehr akzeptabel befunden; vgl. dazu u.a. die mittlerweile zum Klassiker avancierte Arbeit von Joseph W. Trigg: Origen. London 1934, S. 34 u.ö.

38 In dieser Form überliefert bei Simpl. in Cael. 292 b 24. 


\section{4. '́топі́ $\alpha$ als Unsinn oder Konventionsbruch: Kaiser Julian und Heraklit}

Kaiser Julian nimmt Mitte des vierten Jahrhunderts den Faden dort auf, wo ihn die christlich-pagane Debatte bereitliegen hatte: ${ }^{39}$ Mythen erzählen heißt soviel wie „einen instabilen Kompromiss zwischen Wahrheit und Lüge“40 $\mathrm{zu}$ finden, sagt er in einer bemerkenswerten mythentheoretischen Passage seiner Schrift Gegen den Kyniker Herakleios. Dabei ist der Wortlaut der Mythen Lüge, der psychagogische oder moralisch Einsicht stiftende Sinn dagegen wahr. Julians Definition vom mythischen Wortlaut als „Schattenspiel“41 einer strahlenden Wahrheit, deren intensives Licht die meisten Menschen ihrer fehlenden Geisteskraft oder Initiation wegen nicht direkt anzusehen vermögen, geht einerseits via die Schattenspielmetapher des Höhlengleichnisses auf die Vorlage der als Mythos erzählten pia fraus ${ }^{42}$ in Platons Politeia ( $414 \mathrm{bc}$ ) zurück, mit deren

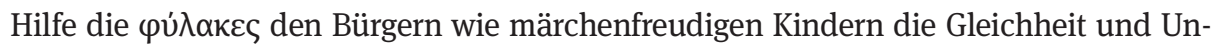
gleichheit des politischen Systems aitiologisch erklären sollen. Tatsächlich ist die pia fraus gewissermaßen eine narrative Auflösung und verschärfende Vereinfachung des Platonischen Aufbaukonzepts für die politische Gemeinschaft - Platonismus fürs Volk

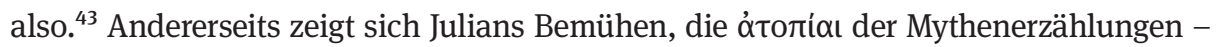
und damit die Anstößigkeiten, wie sie Origenes als Argument ins Feld führte - von vornherein ins Leere laufen zu lassen: ${ }^{44}$ Die $\alpha$ to $\pi_{i} \alpha ı$ sind natürlich Lügen ( $\left.\psi \varepsilon v \delta \tilde{\eta}\right)$, und wo die Mythen die Wahrheit sprächen, seien sie frei von jeder $\alpha$ толí $\alpha$. Mythen haben somit den Charakter eines Rätsels ( $\alpha$ ı̌vı $\mu \alpha):{ }^{45}$ Solange man am Wortlaut bleibt, ergibt sich nur Unsinn, sobald man den Wortlaut untergeht und gewissermaßen hinter sich bringt, eröffnet sich ein ganz anderes Verständnis, es eröffnet sich eine Welt. In dieser Struktur, das heißt in der Rätselhaftigkeit der Rätsel und in der Tatsache, dass Rätsel überhaupt gestellt werden, liegt aber durchaus eine unbestreitbare Sinnhaftigkeit. Ähnlich wie bei einem Witz eröffnet sich nämlich im Moment des Begreifens schlagartig

39 Im Folgenden nach der Schrift Jul. or. 7 (Gegen den Kyniker Herakleios).

40 Maria Carmen De Vita: Giuliano imperatore filosofo neoplatonico. Mailand 2011, S. 108 (mit Hinweis auf die Quelle bei Aelius Theon prog. 3).

41 De Vita (s. Anm. 40), S. 108 (mit Anm. 143) und Jul. or. 7, 206c-207c.

42 Julian selbst spricht von einem Lügengebilde, das sich nach der Notwendigkeit der Seelenanleitung des Lesers richtet (De Vita [s. Anm. 40], S. 107 und Anm. 138: Jul. or. 7, 205c).

43 Vgl. dazu Malcolm Schofield:The Noble Lie. In: G. R. F. Ferrari: The Cambridge Companion to Plato’s Republic. Cambridge 2007, S. 138-164.

44 Aber bei Julian gilt immerhin: Die Mythen sind an Rituelles rückgebunden, sie haben ,einweisenden" Charakter (sie sind $\tau \varepsilon \lambda \varepsilon \sigma \tau$ “ der Mythopoiie tut man Gottes Werk (mit).Vgl. zur Theurgie auch im christlichen Kontext beispielsweise Stock (s. Anm. 28), S. 152-176, und Charles Stang: Apophasis and Pseudonymity in Dionysius the Areopagite: „No Longer I“. Oxford 2012, S. 105-116.

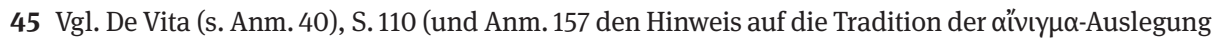
bei Plutarch und Origenes). 
eine ganze stimmige Welt. Auch dieser Gedanke ist in der Tradition gut verbürgt. ${ }^{46}$ Und es ist ein Gedanke, der nach Julian sozusagen die Nagelprobe für die „Echtheit“ des Mythos vorgibt: Eine Erzählung, die keinen welterschließenden hinterweltlichen Sinn aufzuweisen hat, wo der Groschen nicht in einer sinnerschließenden Einsicht fällt, ist kein Mythos, sondern bleibt bloße Lüge, ein Schattenspiel ohne Verweis auf die Lichtquelle, ähnlich wie ein Rätsel oder ein Witz ohne Pointe. In seiner Entgegnung auf einen Kunstmythos des Kynikers Herakleios erhebt Julian genau diesen Vorwurf: Herakleios habe eine bunt ausstaffierte seltsame Geschichte produziert, die keinen philosophischen Erklärwert besitze - und die deswegen schlicht als obszön zu gelten habe. Julian argumentiert hier in der Sache also ähnlich wie Origenes.

In diesem Sinne zitiert Julian dann auch das berühmte Diktum des alten Heraklit von

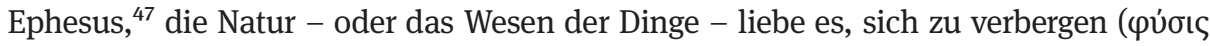

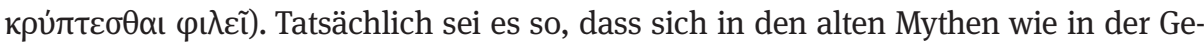
samtwirklichkeit das Wesen der Dinge gleichzeitig zeige und verberge, es sei „hidden in plain view“. Der bei Sallust in De dis et mundo vorfindbare Gedanke, dass sich die Wahrheit in den Mythen als so vertraut menschlich und doch so verstörend nichtmenschlich, so offensichtlich überall und doch so geheimnisvoll, so präsent und gleichzeitig so realitätsenthoben, so schlicht überzeugend und gleichzeitig so wirklichkeitsfremd darbietet, wird mit dem ebenfalls aus Sallust bekannten Gedanken verbunden, die gesamte Welt sei als ein einziger, positiv änigmatischer Mythos anzusehen.

Dazu passt dann im Übrigen auch das Wort, das bei Julian an die systematische

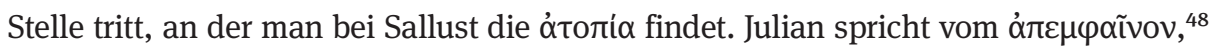
das heißt das „Unangemessene“, „Unpassende“, das „Misfit“, naiv etymologisierend ganz im Sinne des eben zu Julian Geäußerten: das, was in der Erscheinung oder Auftretensweise seinem Inhalt entgegensteht oder nicht mit ihm zu kongruieren scheint.

\section{5. ótomí $\alpha$ als Ortlosigkeit: Porphyrios und noch einmal Julian}

Sallust hatte es damit auf den Punkt gebracht, dass er den Mythos mit der Welt als ganzer verglich, statt ihn mit einer Sage aus vergangener Zeit gleichzusetzen: Diese Dinge, die hier erzählt werden, die átoríaı eingeschlossen, sind nie und nirgends geschehen, doch

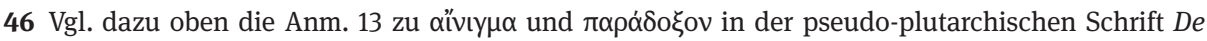
Homero (II) 92.

47 Die Neuplatoniker zitieren Heraklit mit großer Häufigkeit und zahlreiche Heraklitzitate sind uns überhaupt nur durch Schriften der Platoniker bekannt. Im Falle von Julians Mythentheorie in or. 7 kommt aber noch hinzu, dass er seinem Argumentationsgegner, dem Kyniker Herakleios, den besseren Philosophen, den alten Herakleitos aus Ephesos entgegenhält. Dies geschieht nicht nur durch direkte Zitate, sondern auch durch die Häufung typisch „herakliteischer“ Motive wie eben dem Motiv des Rätsels in der Welterschließung (vgl. dazu etwa Heraklit fr. B56, B34 und B93).

48 Vgl. De Vita (s. Anm. 40), S. 111-114; Jul. or. 7, 219a; 217cd; 8, 170ab. 


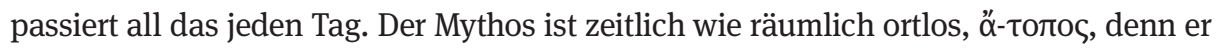
erzählt den Lauf der Welt, nicht den Verlauf einer Geschichte.

Hier gerät man vielleicht an den Kern der eigentlichen Kontroverse von christlichen und paganen Platonikern über den Wert von Mythen. Es geht nicht um einzelne

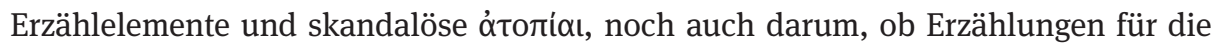
Wahrheit überhaupt etwas taugen. Es geht, so scheint es, um das Bezugsobjekt des Erzählten als raumzeitlicher Gegenstand. Repräsentativ für die christliche Auffassung dürfte die Aussage des Zweiten Petrusbriefs $(2$ Petr 1,16) stehen: Nicht auf ausgeklügelt

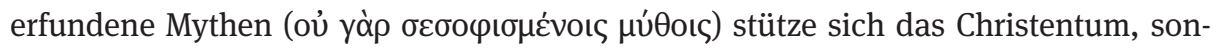
dern auf das Augenzeugnis eines geschichtlich greifbaren göttlichen Machterweises, also auf einmalige historische Tatsachenerfahrungen. Gegen diesen Gedanken der geschichtlichen Parusie in der christlichen Auffassung protestiert Kaiser Julian in seinem Helios-Hymnos, und es ist einem dabei, als würde er hier ganz gezielt gegen den Johanneischen Logos-Hymnos angehen: Die Sonne ist das, was man zu allen Zeiten also ungeschichtlich und keinesfalls einmalig - gesehen und zum normativen Ausrichtungspunkt des Lebens und der Zeiten gemacht hat, ${ }^{49}$ Christus dagegen habe von den noch Lebenden keiner jemals gesehen - ein schwerwiegendes Problem für das

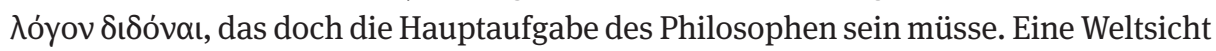
von mythischer Zeitlosigkeit und eine Philosophie des semper, ubique, ab omnibus wird hier der historischen Versicherung „wir haben Seine Herrlichkeit gesehen“ (Joh 1,14) und der Theologie des in illo tempore wirksam entgegengesetzt. Die átoтí findet sich in dieser Betrachtungsweise geradezu positiv gewendet zu einer Ubiquität, die zur Welterklärung besser taugt als ein zeitlich isolierbares Einzelereignis. Der erzählte Mythos ist für Julian die narrativ aufgelöste Grammatik des Kosmos. Gerade als Erzählung eines räumlich wie zeitlich ortlosen Zustands der Welt wird nach Julians These der Mythos also kosmo-logisch in dem von Platon geforderten Sinne. Wie der Mythos in diesem kosmologischen Sinne gesehen als Form göttlicher Selbstmitteilung dem Weltbau gleichgestellt werden kann oder vielmehr der Kosmos (wie bei Sallust gesehen) als ein einziger Mythos verstanden werden darf, belegt nicht zuletzt das zunächst einmal naiv romantisch anmutende Datum, dass die Griechen in den Gestirnkonstellationen ewig wiederkehrende Bebilderungen ihrer Mythen erkannten.

Der einzige vollständig erhaltene Text einer allegorisierenden Auslegung eines (Stücks aus einem) Mythos durch einen führenden Platoniker ist m.W. die um 304/305

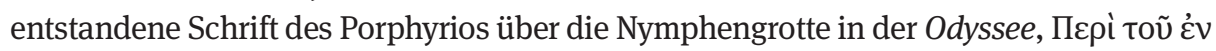

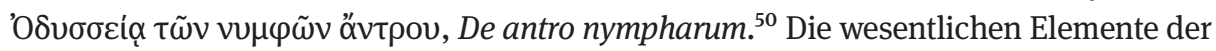

49 Der Gedanke verweist zurück auf Plat. pol. 508a-e.

50 Maßgebliche Edition ist bis auf Weiteres immer noch die von L. G. Westerink: Porphyry, The Cave of the Nymphs in the Odyssey. Buffalo 1969; neuer: Robert Lamberton: Porphyry, On the Cave of the Nymphs. Barrytown, N.Y. 1983. Zur Singularität und Bedeutung des Textes als einziger im Ganzen erhaltener neuplatonischer Traktat der Mythenallegorisierung vgl. z. B. Katell Berthelot: Philo and the Allegorical Interpretetaion of Homer in the Platonic Tradition (With an Emphasis on Porphyry's De 
kanonisch-platonischen Mythenauslegung lassen sich anhand dieser kleinen Auslegungsschrift allesamt dingfest machen und überprüfen. Den Anfang macht genau die Feststellung des Autors, die von Homer beschriebene Höhle oder Grotte gebe es geographisch in Ithaka gar nicht, und diese óto $i \dot{\alpha} \alpha$ als reale Ortlosigkeit setze sich darin fort, dass die Beschreibung der Höhle eigentlich zur Erzählung nichts beizutragen scheint und einem deshalb seltsam fehl am Platz vorkommen müsse (Porph. antr. 2). ${ }^{51}$ Das stoße den aufmerksamen Leser darauf hin, dass sich hier in Wahrheit eine Beschreibung der

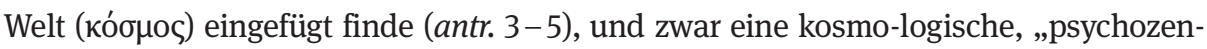
trische“ Beschreibung, die dazu angetan ist, zu zeigen, wie sich die zyklische Bewegung der Seelen vom Göttlichen und von den Göttern zu den Seelen im Wirklichkeitsaufbau und als Wirklichkeitsaufbau begreifen lässt (v.a. antr. 7 und 9: „ein Symbol aller unsichtbaren Mächte“, und von da an passim). ${ }^{52}$ Doch das muss dafür an dieser Stelle genügen.

\section{6. '́xoría als Unnatürlichkeit: Augustinus und Athanasius (und C. S. Lewis)}

Ein kurzer und durchaus eklektischer Blick auf die Einschätzung christlicher Platoniker soll diese Ausführungen abschließen. Wie bereits gesagt: Der Hauptstreitpunkt der allegorisierenden Interpretation welterklärender Erzählungen scheint vor allem in der Bedeutung der Frage zu liegen, ob das Erzählte als historisch verortbar angenommen werden darf. In diesem Sinne nahmen die christlichen Denker die Bibelgeschichten als historisch und symbolisch an, eben als realsymbolisch im Sinne der Symboldefinition Walter Benjamins: als Anschauung des Universalen im greifbaren Einzelvorkommnis. ${ }^{53}$ Der platonischen Mythenauslegung erschien dieser Partikularaspekt dagegen als zu vernachlässigen und schließlich als zu überwinden, als pädagogisches $\psi \varepsilon \varepsilon \tilde{\delta} \delta$, , denn in diesem historisch-partikularen Sinne eines Einzelvorkommnisses war das Erzählte nie

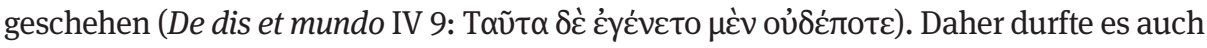

antro nympharum). In: Maren Niehoff (Hg.): Homer and the Bible in the Eyes of Ancient Interpreters. Leiden/Boston 2012, S. $155-174$, v. a. S. 165.

51 Dieses Interesse am geographischen Realwert des bei Homer Erzählten und dem Wert des Literalsinns des Mythos ist bei all dem unbestreitbar. In diesem Sinne ist es auch richtig, dass „the allegorical meaning attributed to the description of the cave does not discredit the literal meaning nor does it empty its significance. On the contrary, it seems that Porphyry, and the Neo-Platonists in general, attached importance to the literal meaning and to the historical or geographical exactness of the texts they interpreted“ (Berthelot [s. Anm. 50], S. 168).

52 Es fehlt zur Untermauerung dieser psychozentrischen Weltdeutung selbstverständlich auch nicht der Hinweis darauf, dass Platon im Höhlengleichnis das Schicksal der Seele im Kosmos ebenfalls sehr adäquat mit dem Bild einer Höhle beschrieben habe (Porph. antr. 8).

53 Vgl. Walter Benjamin: Der Ursprung des deutschen Trauerspiels. Frankfurt 1978, S. 140 -141, sowie die Einführung von Pablo Maurette zu seiner Übersetzung: Porfirio, El antro de las ninfas en la Odisea/ Puntos de partida hacia los inteligibles. Buenos Aires 2007, S. 17-27. 
ruhig als anstößig wirken, solange es nur zum Denken anzustoßen vermochte. Generell gilt ja, dass was im Leben als störend wirkt, in der Philosophie als aufstörend wirkt, nämlich für das Nachdenken.

Der Unterschied in der Auffassung lässt sich vielleicht ganz gut anhand der christlichen Deutung der biblischen Wundergeschichten im Kontrast zu den paganen mythischen Geschichten dokumentieren: Von Wundern, so meinen die christlichen Platoniker, sollte man sich wünschen können, dass sie einem wirklich passierten. Von dem, was von Skylla, Orion, Arethusa und Daphne und anderen wundersamen Vorgängen in den paganen Mythen erzählt wird, kann man sich nicht wünschen, dass es einem wirklich - historisch - passiere. Hier kann man tatsächlich nur darauf hoffen, dass solches sich nie ereignet hat. Und so sagt Augustinus, dass ein Wunder unsere Natur weder vergewaltigt noch ihr überhaupt in irgendeiner Weise entgegensteht, sondern sie in eigener Weise komplettiere: „Ein Wunder (portentum) geschieht nicht wider die Natur, sondern wider die bekannte Weise der Naturabläufe“ (Aug. civ. XXI 8,35; dort auch die Erklärung: portentum heiße das Wunder, weil es etwas anzeigt oder auf etwas vorverweist, praeostendo).

Warum das mit der christlichen Theologie zusammenpasst, in der der universale Logos der Welt historische Wirklichkeit wird, erklärt Athanasius in seinen Predigten über die Auferstehung, wenn er sagt, Christus habe Menschengestalt angenommen und als Mensch gelebt, damit die, welche ihn als Schöpfer des gesamten Kosmos anerkennen, aus seinen Werken hier auf Erden erkennen können, dass in diesem Menschenleib das Wort Gottes wohnte. ${ }^{54} \mathrm{C}$. S. Lewis hat in seinem (in einigen Facetten freilich vielleicht durchaus verwirrenden) Essay Miracles diese Bemerkung des Athanasius so ausgedeutet: ${ }^{55}$ Christus, wahrer Mensch und wahrer Gott, wirkte als historischer Mensch dasselbe wie als Gott, der alle Naturgesetze eingerichtet hat. So tat Jesus in historischer Einmaligkeit dasselbe wie der Schöpfer im immerwährenden Hervorbringen und Bewahren der Schöpfung, nur als Mensch, und das wird - anders als im Schöpfungsablauf als ganzem - als Wunder begriffen, also als Ereignis, das als historisch isolierbarer Ablauf in Staunen versetzt, oder als ein Ereignis, das eine im Normalzusammenhang des Geschehens frappierende, zunächst unerklärbar erscheinende Anomalie beschreibt, obwohl, oder gerade weil es ein historischer Beleg dieses Normalzusammenhangs ist. Jesus tut also vor den Augen seiner historischen Mitmenschen das, was sie in gewöhnlicher Weise aus den Schöpfungsregeln und Schöpfungsabläufen kennen, aber eben in außerordentlicher, einmalig greifbarer Weise. Damit bricht er nicht mit der Natur, er übertrumpft sie auch nicht, er setzt sie nicht außer Kraft, sondern er vollendet sie spektakulär im Einzelnen. Als Beispiel nimmt Lewis die Wundererzählung von der Weinverwandlung: Dass sich Wasser in Wein verwandelt, ist nichts Spektakuläres, jeder weiß, dass das andauernd und überall auf der Welt geschieht: Damit er Trauben trägt

54 Athan. incarn. (PG XXV, 96-198), I 18, vgl. den gleichen Gedanken in VI 43 und VII 49.

55 Der Essay Miracles war ursprünglich (1947) als eigenständige Broschüre erschienen, bildete danach aber das Kapitel 2 von C. S. Lewis: God on the Dock. Essays in Theology and Ethics. Grand Rapids 1970, S. 25-36. 
und damit diese Trauben gepresst werden können, muss man den Weinstock wässern. Das Zugeführte Wasser wird durch natürliche Abläufe und durch das Zutun des Menschen (Keltern, Lagern etc.) zu Wein. Wein ist „die Frucht des Weinstocks und der menschlichen Arbeit“, wie eine liturgische Zeile es sagt. Jesus vollführt genau diesen Regelablauf des Verwandelns von Wasser, das bereitsteht, unter menschlichem Zutun (nämlich seinem eigenen Wirken) in Wein. Nur eben instantan. Und dass der Wein besonders gut ist, zeigt, dass hier auf Vollendung von etwas Natürlichem abgezielt wird. Insofern ist das Wunder ein Zeichen für das Wirken Gottes.

So ist das Wunder in christlicher Sicht keineswegs ein Bruch mit den natürlichen, bekannten Abläufen, sondern eine bedeutsame Raffung und momentane Vollendung der Abläufe, die der Schöpfer seiner Schöpfung zugedacht hat. Eine christliche Wundererzählung entspricht weder der Intention noch dem Genre nach einem antiken Mythos, sondern sie berichtet von etwas, das jeder als natürlich gerne akzeptiert, und was in der gedrängten Intensivform, in der es sich als Wunder ereignet, historisch als zeichenhafte Vollendung der Natur spektakulär offenbar wird. Das Wundersame liegt mithin darin, dass etwas Natürliches und als solches Bekanntes sich in besonderer Weise ereignet und dieses Besondere oder diese Weise als das Wirken von etwas verstanden wird, das souverän über der Natur steht, dieser Natur aber deswegen noch lange nicht entgegensteht. Wunder sind daher nichts Widernatürliches, auch nichts Unnatürliches, ihre Außerordentlichkeit liegt nicht darin, dass sie aus der Ordnung der Welt ausscheren, sie sind vielmehr ein punktuelles Aufzeigen der Vollendungsgestalt des Natürlichen. Die Belege dafür aus den Schriften der alten christlichen Theologen ließen sich häufen, als pars pro toto stehe aber lediglich folgende Aussage des Augustinus:

Gott wirkt auch die Blitze und Donner gewöhnlicher Art. Weil aber auf dem Berg Sinai dergleichen in ungewöhnlicher Weise sich ereignete [ ... und] aus bestimmten Merkmalen sich ergab, dass durch dieselben gewisse Zeichen gegeben würden, so waren es Wunder. (Aug. trin. III 5,38)

Konsequenzen daraus waren erstens: Die Erklärung von Wundern, portenda oder miracula, als gerafften und über sich hinausweisenden Vollendungsabläufen lässt auch wahre Wunder von dem trennen, was die mittelalterliche Tradition als monstrositas vom miraculum unterschied. Eine solche historische monstrositas tut genau das, was die mythischen ótotíaı zu tun drohen, wenn sie als „wirklich wahr“ angenommen würden: Sie würden den Rahmen des für den Menschen (in platonischer Sicht) Akzeptablen sprengen. Denn:

It cannot be too strongly insisted that a world which is to be a moral order must be a physical order characterized by law or regularity. [...] The theist is only concerned to invoke the fact that lawabidingness [...] is an essential condition of the world being a theatre of moral life. Without such regularity in physical phenomena there could be no probability to guide us: no prediction, no prudence, no accumulation of ordered experience, no pursuit of premeditated ends, no formation 
of habit, no possibility of character or culture. Our intellectual faculties could not have developed. $^{56}$

Die Deutung des Augustinus zeigt, dass sich ein Wunder eben tatsächlich unter anderem dadurch auszeichnet, dass der Mensch es sich für sein eigenes Leben herbeiwünschen können muss, dass es eine positive Bedeutsamkeit im Sinne eines Vollendungsvorgriffs hat, die ein Menschenleben gleichwohl nicht vollkommen und natursprengend verändert. Es ist ein Zeichen innerhalb eines naturentsprechenden Regelverhaltens und mehr noch: auch ein Hinweiszeichen für dieses Verhalten. Das unterscheidet das Wunder etwa von den oben beispielhalber genannten Metamorphosen-Mythen zu Skylla oder Daphne wie bei Ovid, so wunderbar sie auch anmuten mögen. Ein Wunder muss, so könnte man vielleicht sagen, in der First-person-perspective (positiv) annehmbar sein.

Zweitens: Wunder können, so hat sich daraus schon ergeben, als Zeichen verstanden werden, als on $\mu$ ĩov oder signum (wie im Hendiadyoin „Zeichen und Wunder“). Zeichenhaft ist aus christlicher theologischer Perspektive das Handeln durch Wunder in Übereinstimmung mit Gottes Plan für die Welt, die sich hier besonders intensiv und bedeutsam historisch kundtut. Hier tut jemand - der Wundertäter als Өcoupyós - Gottes Dinge, die Vollendungsgestalt des Schöpfungsplans blitzt hier in einem einzelnen Geschehnis oder in einem individuellen Menschen auf und macht das Wirken Gottes an und in der Welt, insbesondere sein Heilswirken, somit transparenter. Von ó толí keine Spur.

\section{Fazit}

Der Kampf um Mythos und Mythendeutung im Platonismus des christianisierten Kaiserreichs lässt sich somit in seinen Hauptpunkten auf einige wenige Fragen zusam-

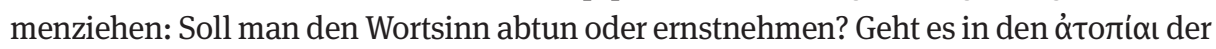
Mythen um ein als raumzeitlich ortlos allegorisierbares Symbol oder um ein Realsymbol? Bis zu welcher Grenze ist der Mythos (psychagogischer) Anstoß und ab wann ist er (,atopische“) Anstößigkeit? Bringt oder bedingt der Mythos ein Abstoßen (nach „oben“), weil er abstoßend ist? Und wenn ja: Bis zu welcher Grenze darf man diese Dialektik in theoretischer Redlichkeit annehmen, bis zu welcher Grenze ist sie für moralische Redlichkeit annehmbar? Die Frage ist damit letztlich nicht: Ist der Mythos wahr? Denn so kann sie nicht beantwortet werden. Die Frage ist: Geht die Wahrheit im Mythos auf?

56 So F. R. Tennant: Philosophical Theology, Bd. 2: The World, the Soul, and God. Cambridge 1937, S. $199 \mathrm{f}$. Ausführlicher zum gleichen Gedanken Friedrich Hermanni: Metaphysik. Versuche über letzte Fragen. Tübingen 2011, S. 138. 
